\title{
Forecasting the Anti-Rabies Vaccine Demand at Jawaharlal Medical College and Hospital, Ajmer, Rajasthan: A Comparative Analysis based on Time Series Model Renu Bedi, Narendra Verma, Kunal Gautam, Varun Agiwal
}

${ }^{1}$ Professor \& Head, Department of Community Medicine, Jawaharlal Nehru Medical College, Ajmer, Rajasthan, India; ${ }^{2}$ PG, Department of Community Medicine, Jawaharlal Nehru Medical College, Ajmer, Rajasthan, India; ${ }^{3}$ Senior Demonstrator, Department of Community Medicine, Jawaharlal Nehru Medical College, Ajmer, Rajasthan, India; ${ }^{4}$ Senior Lecturer, Indian Institute of Public Health, Hyderabad, India

\begin{tabular}{|c|c|c|c|c|c|c|c|}
\hline Abstract & Introduction & Methodology & Results & Conclusion & References & $\underline{\text { Citation }}$ & Tables/Figures \\
\hline
\end{tabular}

\section{Corresponding Author}

Dr. Varun Agiwal, Senior Lecturer, Indian Institute of Public Health, Hyderabad, Telangana - 500033 India

E Mail ID: varunagiwal.stats@gmail.com

\section{Citation}

Bedi R, Verma N, Gautam K, Agiwal V. Forecasting the Anti-Rabies Vaccine Demand at Jawaharlal Medical College and Hospital, Ajmer, Rajasthan: A Comparative Analysis based on Time Series Model. Indian J Comm Health. 2021;33(3):451455. https://doi.org/10.47203/IJCH.2021.v33i03.008

Source of Funding: Nil Conflict of Interest: None declared

\section{Article Cycle}

Received: 03/05/2021; Revision: 21/07/2021; Accepted: 05/09/2021; Published: 30/09/2021

This work is licensed under a Creative Commons Attribution 4.0 International License.

\section{Abstract}

Background: In India, high mortality and morbidity rates of human rabies is observed. Hence, a structured surveillance system is yet to be put in place for public health discussion. At the tertiary care hospital and all public health centres, requirement of anti-rabies vaccine is needed in advance to predict the upcoming months coverage so that wastage of vaccine is minimum. Objective: To find a suitable model for forecasting the appropriate stock of anti-rabies vaccines to avoid shortage and oversupply at anti rabies clinic. Methods and Material: This was a record based cross sectional study, conducted at anti rabies clinic of Jawaharlal Nehru Medical College and Hospital, Ajmer. Data of used anti rabies vaccine was taken from immunization inventory during the period from 2017 to 2020. Time series analysis based on Holt-Winter and Box-Jenkins methods were carried out to predict the need of vaccine. Results: Study series was not stationary and stationarity was observed by taken difference in the observation between two consequent months. Residuals of the series were normally distributed and independent to each other. $\operatorname{ARIMA}(0,1,1)$ was the best model in comparison to Holt-Winter model for prediction because of low value of model selection criterion. The forecasted value for anti-rabies vaccine was done for the year 2021. Conclusions: The following study concluded that time series can be used as a tool to forecast anti-rabies vaccine coverage and will help the policy makers to formulate appropriate plans and strategies and improve the management of vaccination resources and inventory.

\section{Keywords}

Anti-rabies vaccine; Time series; Model selection criterion; Rabies; Vaccination

\section{Introduction}

Rabies is a zoonotic viral disease that is spread from animals to other animals and to humans through their saliva. It is a major public health problem worldwide as well as in India because 59,000 people and 20,000 people die from rabies every year globally and India respectively(1,2). In India, major observed sources of morbidity and mortality due to rabies, are dog bite followed by cats and other animals(3). A significant proportion of cases are observed in children and elders, despite the availability of safe and effective vaccines(4). Lack of awareness regarding preventive measures against rabies and post-exposure prophylaxis, and insufficient vaccination of animals are the leading causes for rabies cases(5). In India, the primary healthcare settings have irregular supply of anti-rabies vaccine and immunoglobulins to be responsible for the same(6). The National Rabies Control Program is rolled out by the government of India (2013), with an objective that human deaths due to rabies should be eliminated and break down the transmission chain of rabies through canine (dog) wild rabid animals. This programme focuses on training of health care professionals about management of animal bites, providing post exposure prophylaxis, creating awareness and reduction of animal bites, vaccination and 

sterilization of stray dogs(3). Government of India is taking all necessary actions through National Rabies Control Programme for directing the effective progress of rabies control to achieve the target set by the WHO for global elimination of human deaths from dog-mediated rabies by the year 2030(7). As no effective treatment is available to control the rabies, the target of global elimination can be achieved only through timely and appropriate vaccination.

The amount of anti-rabies vaccines existing in particular health centres such as primary health centres, community health centres and at district-level hospitals during a particular time period is the major issue to achieve the global target. So, the rationale is to guide the inventory and purchase of injection ARV which is also lifesaving and emergency approach of the animal bite treatment. The futuristic model can be utilised for other desired stock and purchases in the institution to add up to the public health facilities. Such studies can also be utilised to explore alternative strategies and contingencies which can be purchased in the hour of need it will also give a foresight for budget allocation and an educational planning. Therefore, there is a need to predict the required amount of anti rabies vaccines. In statistics, regression and time series models are the effective control and prevention method to predict the future observations(8). The major aspect of time series and regression models are to examine the inference of the series by fitting the best preferred model and find out the key feature of process such as linearity, stationarity, trending, normality etc(9). To allow possible unpredictable nature of future observations in time series approach, it is natural to suppose that each observation depends on own present or past values. The auto-regressive integrated moving average and exponential smoothing (Holt-Winters) models are used popularly and are also important tools for forecasting the behaviour over time(10).

\section{Aims \& Objectives}

To estimate the vaccine requirement at anti rabies clinic of the Jawaharlal Nehru Medical College, Ajmer and predict the one year forecast to avoid shortage and oversupply.

\section{Material \& Methods}

A record based, cross sectional study design was considered to obtain the monthly registered data of four years for anti-rabies vaccines during the period from January 2017 to December 2020. The data set for this study was collected from immunization records maintained at anti rabies clinic of Jawaharlal Nehru Medical College, Ajmer. This data set consists the information regarding the number of vaccines injected to the beneficiaries for 48 months of the study period. The month wise data was entered into Microsoft Excel 2013 and analysis was done using $\mathrm{R}$ programming language version 3.3.1. Permission regarding the data collection was taken from Department of Community Medicine and ethical clearance for the study was obtained from the Institutional Ethics Committee of Jawaharlal Nehru Medical College, Ajmer.

An autoregressive integrated moving average ARIMA(p, d, q) $\}$ and exponential smoothing models were considered the best fitted model and then forecast the future demand of the number of anti rabies vaccines. In the ARIMA model, $p$ is the order of autoregressive terms, $d$ is the degree of differencing and $q$ is the order of movingaverage terms. The procedure of Box and Jenkins methodology was introduced to carry out the time series analysis which includes model identification, estimation of model parameters, checking the model accuracy and forecast future values. For independence of residuals, Ljung-Box test statistic was used. Shapiro-Wilk test was also used to observe the normality condition. For checking the stationarity condition, augmented Dickey-Fuller (ADF) test was applied. The plots of autocorrelation function (ACF) and the partial autocorrelation function were drawn to determine the most-likely values of $p$ and $q$. Finally, Akaike information criteria (AIC), root mean square error (RMSE) and mean absolute error (MAE) was used for selecting the best-fitted model and was observed the forecast accuracy.

\section{Results}

During last four years, a total of 38035 doses of anti rabies vaccine were used at anti rabies clinic of Jawaharlal Nehru Medical College \& Hospital, Ajmer. Number of vaccine doses used for beneficiaries were maximum (Median $=760$ ) in year 2017. The highest number of vaccines were used in the month of January (Median $=858$ ) and June (Median = 853) (Table 1)

We identified outlier(s) at first, using box plot because it affects the quality of the forecasting. [Figure 1] describes the presence of outlier(s) in the series by spreading data before and after outlier detection. From [Figure 1], we observed that 5 observations of the data were treated as outliers and were replaced by their median in respect to year wise. The p-value of Shapiro-Wilk test was 0.2741, shows that residuals were normally distributed. For checking the stationarity, Augmented Dickey-Fuller (ADF) Test was used. The Dickey-Fuller statistic is -2.31 with $p$ value 0.45 which indicates that the series is not stationary. To take a first difference between present and past values, Dickey-Fuller statistic is -4.35 with $p$ value 0.01 . Thus, the series is stationary at first difference $(d=1)$. The order of autoregressive ( $p$ ) and order of moving average (q) were determined using autocorrelation function (ACF) and partial autocorrelation function (PACF) as displayed in [Figure 2]. In the [Figure 2], we observed that PACF plot shows a slowly decreasing trend and do not crosses the upper confidence interval so the lag value of AR order is $p$ $=0$, whereas ACF plot crosses the upper confidence interval for the first time after lag 1 so the lag value of MA 
order is $q=1$. This shows that series having dependence based on residuals components only.

For comparison between time series and exponential smoothing models, AIC, R2 and RMSE were calculated and result was recorded in [Table 2]. [Table 2] was concluded that ARIMA model is the best model to forecast the series because the value of AIC and RMSE is minimum in comparison with the exponential smoothing model while $\mathrm{R} 2$ value is 0.46 , shows that $46 \%$ of the data is well explained by the time series model. The Ljung-Box $Q$ was used to test the observations are random and independent and result was recorded in [Table 2]. The Ljung-Box $Q$ was statistically non-significant $(p=0.7989)$ for ARIMA model, indicates that the residuals of the model are independent of each other. Overall, we stated that residuals are independently normally distributed, and series was stationary at first difference.

For understanding the performance of the ARIMA and exponential smoothing models, forecast accuracy was measured and was shown in [Table 3] based on root mean square error (RMSE) and mean absolute error (MAE). The result was concluded that RMSE and MAE were lower for ARIMA model for achieving a better forecast performance of anti rabies vaccine requirement. Thus, the ARIMA model performs well than the exponential smoothing model to forecast ARV vaccination coverage. The forecasted value for the next one year was done using the ARIMA model in [Table 4] and [Figure 3]. We observed that maximum number of vaccine requirement was observed in the month of March 2021, followed by February 2021 and April 2021.

\section{Discussion}

A total of 38035 doses of anti rabies vaccine were utilized from the year January 2017 to December 2020 at anti rabies clinic. This record based cross sectional study was estimated the future requirement of vaccines under two statistical models. It was observed that in the month of January 2017 and June 2017, maximum number of vaccines doses were inoculated to the exposed individuals, that means more animal bite cases was observed in the community. Majority of vaccine was used during the summer season. The major causes during these months are due to more outdoor activities by the people, summer and winter vacations of the children. This type of study was applied on various vaccines like Measles, BCG, OPV etc. based on exponential smoothing model to determine the vaccine requirement (Shastri et al.(10) and Kendre et al.(11)) whereas ARIMA model was considered to find the malaria cases using climatic factors in Delhi and forecasted the number of children at the time of terminal contraception by Kumar et al.(12) and Mumbare et al.(13) respectively.

In our study, we found that series is to be non-stationary and taken a first difference to make the series stationarity. Similar result were taken by Sahisnu et al.(14), Alegado and Tumibay(15) and Mumbare et al.(13) for various vaccines prediction and forecasting the number of children at the time of terminal contraception. According to the results, residuals of our series were independent and normally distributed. These similar results were to be obtained by Paso and Ngamjarus(16) for forecasting the presence of rabies in dogs at Thailand and Kendre et al.(17) for measles vaccine requirement forecasting.

This study concludes that ARIMA model is most suitable for predicting the anti rabies vaccine as compared to exponential smoothing model. Various researchers also compared various models in their study. Alegado and Tumibay(18) indicated that MLPNN model is superior to the ARIMA model for forecasting the monthly vaccine demand. Paso and Ngamjarus(16) obtained the SARIMA model as the best model for predicting the number of dogs with rabies. For analysis purpose, the current study considered only the number of vaccines utilized, but other factors such as population density, density of rabies animals, vaccination rates also equally responsible for the improvement of anti rabies vaccine requirement, which is also a shortcoming of our study.

\section{Conclusion}

A novel strategy procedure was applied to predict the required vaccine stock of anti rabies vaccine. We found that the $\operatorname{ARIMA}(0,1,1)$ model assures the optimum supply of vaccine and provides a better decision for policy makers in improving vaccination coverage. The forecast value for the year 2021 shows that forecast interval includes the observed value for the month of January-April, 2021. The difference in required doses for the tertiary care centre always depends on the epidemic logical scenario including the services/logistic provided at other peripheral health centre.

\section{Recommendation}

As per the study results, we recommend that training of health care professionals about management of vaccine is an important task to reduce the vaccine wastage so that there is no health crisis of vaccine in the community. Each and every person can easily assess the vaccine when it is needed.

\section{Limitation of the study}

Future study includes explanatory variables like population density, growth rate of domestic animals, number of vaccinated animals etc. to improve the appropriateness of the best model. Other appropriate statistical models and techniques should also be considered for the better outcomes of the results.

\section{Relevance of the study}

The significance prospective of the study is that every hospital is stored a sufficient number of vaccine doses in future so that vaccine wastage is optimum level and utilization is more. No need to store extra vaccine in a particular hospital. 


\section{Authors Contribution}

RB: Substantial contributions to conception. NV: drafting the article or revising it critically for important intellectual content. KG: Study design and acquisition of data. VA: Analysis and interpretation of data, final approval of the version

\section{References}

1. WHO. WHO Expert Consultation on Rabies: Third Report; AbelaRidder, B., Ed.;World Health Organization: Geneva, Switzerland, 2018; ISBN 9789241210218.

2. Hampson K, Coudeville L, Lembo T, Sambo M, Kieffer A, Attlan M, Barrat J, Blanton JD, Briggs DJ, Cleaveland S, Costa P. Global Alliance for Rabies Control Partners for Rabies Prevention. Estimating the global burden of endemic canine rabies. PLoS Negl Trop Dis. 2015;9(4):1-20. Available from: https://doi.org/10.1371/journal.pntd.0003709

3. Ministry of Health and Family Welfare, (MOHFW). Draft National Action Plan for Eliminating Dog Mediated. Rabies from India. National Centre for Disease Control, Directorate General of Health Services, Ministry of Health and Family Welfare, Government of India; 2020.

4. Mani RS, Anand AM, Madhusudana SN. Human rabies in India: an audit from a rabies diagnostic laboratory. Tropical Medicine \& International Health. 2016;21(4):556-63. Available from: https://doi.org/10.1111/tmi.12669

5. Kole AK, Roy R, Kole DC. Human rabies in India: a problem needing more attention. Bulletin of the World Health Organization 2014;92 (4) :230. Available from: http://dx.doi.org/10.2471/BLT.14.136044

6. Kapoor P, Baig VN, Kacker S, Sharma M, Sharma M. A crosssectional study of knowledge regarding rabies among attendees of anti-rabies clinic of a teaching hospital, Jaipur. Journal of Family Medicine and Primary Care. 2019;8(1):194-98. Available from: https://doi.org/10.4103/jfmpc.jfmpc 205_18

7. United Nations. Sustainable Development Knowledge Platform: Goal 32015 (2015). Available from: https://sustainabledevelopment.un.org/sdg3

8. de Figueiredo A, Johnston IG, Smith DM, Agarwal S, Larson HJ, Jones NS. Forecasted trends in vaccination coverage and correlations with socioeconomic factors: a global time-series analysis over 30 years. The Lancet Global Health. 2016;4(10):e726-
[Forecasting the Anti-Rabies...] | Bedi R et al 35. Available from: https://doi.org/10.1016/S2214109X(16)30167-X

9. Dougherty C. Introduction to econometrics. Oxford University Press, USA; 2016.

10. Shastri S, Sharma A, Mansotra V, Sharma A, Bhadwal A, Kumari M. A study on exponential smoothing method for forecasting. International Journal of Computer Sciences and Engineering. 2018;6(4):482-5. Available from: https://doi.org/10.26438/ijcse/v6i4.482485

11. Kendre VV, Mumbare SS, Dixit JV, Wadagale AV. Estimation of vaccine requirement by using time series analysis. Medpulse International Journal of Community Medicine. 2019;9(3) :57-63.

12. Kumar V, Mangal A, Panesar S, Yadav G, Talwar R, Raut D, Singh S. Forecasting malaria cases using climatic factors in Delhi, India: a time series analysis. Malaria research and treatment. 2014;1-6. Available from: https://doi.org/10.1155/2014/482851

13. Mumbare SS, Gosavi S, Almale B, Patil A, Dhakane S, Kadu A. Trends in average living children at the time of terminal contraception: a time series analysis over 27 years using ARIMA $(p, d, q)$ nonseasonal model. Indian Journal of Community Medicine. 2014 Oct;39(4):223-228. Available from: https://doi.org/10.4103/0970$\underline{0218.143024}$

14. Sahisnu JS, Natalia FR, Ferdinand FV, Sudirman S, Ko CS. Vaccine prediction system using ARIMA method. ICIC Express Letters, Part B: Applications. 2020;11(6):567-75. Available from: http://dx.doi.org/10.24507/icicelb.11.06.567

15. Alegado RT, Tumibay GM. Forecasting measles immunization coverage using ARIMA model. Journal of Computer and Communications. 2019;7(10):157-68. Available from: https://doi.org/10.4236/icc.2019.710015

16. Paso A, Ngamjarus C. Forecasting rabies in dogs in Thailand: Time series analysis. Naresuan University Journal: Science and Technology (NUJST). 2020;28(4):64-74. Available from: https://doi.org/10.14456/nujst.2020.37

17. Kendre VV, Dixit JV, Bahattare VN, Wadagale AV. Forecasting measles vaccine requirement by using time series analysis. J Evolution Med Dent Sc. 2017;6:2329-33.

18. Alegado RT, Tumibay GM. Statistical and machine learning methods for vaccine demand forecasting: A comparative analysis. Journal of Computer and Communications. 2020;8(10):37-49. Available from: https://doi.org/10.4236/jcc.2020.810005

\section{Tables}

TABLE 1 FIVE NUMBER SUMMARY OF ANTI RABIES VACCINE USED IN YEAR AND MONTH

\begin{tabular}{|c|c|c|c|c|c|c|}
\hline & Variable & Min & $1^{\text {st }}$ quartile & Median & $3^{\text {rd }}$ quartile & Max \\
\hline \multirow[t]{4}{*}{ Year } & 2017 & 522 & 696 & 760 & 846 & 1167 \\
\hline & 2018 & 549 & 599 & 758 & 599 & 1787 \\
\hline & 2019 & 232 & 336 & 426 & 336 & 2015 \\
\hline & 2020 & 404 & 574 & 701 & 574 & 1391 \\
\hline \multirow[t]{12}{*}{ Month } & January & 404 & 513 & 858 & 1379 & 2015 \\
\hline & February & 689 & 702 & 716 & 940 & 1585 \\
\hline & March & 584 & 616 & 668 & 880 & 1391 \\
\hline & April & 445 & 633 & 717 & 788 & 934 \\
\hline & May & 547 & 674 & 743 & 1002 & 1702 \\
\hline & June & 509 & 739 & 853 & 1020 & 1411 \\
\hline & July & 406 & 681 & 775 & 824 & 963 \\
\hline & August & 360 & 482 & 548 & 661 & 921 \\
\hline & September & 338 & 538 & 608 & 709 & 1003 \\
\hline & October & 232 & 551 & 704 & 811 & 992 \\
\hline & November & 296 & 422 & 561 & 940 & 1787 \\
\hline & December & 331 & 422 & 521 & 855 & 1652 \\
\hline
\end{tabular}




\section{TABLE 2 SELECTING BEST MODEL BASED ON MODEL STATISTICS}

\begin{tabular}{|c|c|c|c|c|c|}
\hline Model & AIC & Rodel Fit Statistics & RMSE & Statistics & P-value \\
\hline ARIMA(0,1,1) & 594.0512 & 0.4675 & 225.8699 & 1.1824 & 0.7989 \\
\hline Exponential Smoothing & 620.9561 & 0.0964 & 246.4697 & 30.6137 & 0.0001 \\
\hline
\end{tabular}

TABLE 3 ACCURACY MEASURE TO SELECT THE BEST MODEL FOR FORECASTING PURPOSE

\begin{tabular}{|c|c|c|}
\hline Model & \multicolumn{2}{|c|}{ Forecast Accuracy } \\
\hline ARIMA (1,0,1) & RMSE & MAE \\
\hline Exponential smoothing & 257.5948 & 195.6627 \\
\hline
\end{tabular}

\begin{tabular}{|c|c|c|c|c|}
\hline Month-Year & Forecasted Values & Lower $95 \% \mathrm{Cl}$ & Upper $95 \% \mathrm{Cl}$ & Observed \\
\hline Jan-21 & 1101.14 & 969.10 & 1533.18 & 1340 \\
\hline Feb-21 & 1316.71 & 1031.37 & 1602.05 & 1350 \\
\hline Mar-21 & 1404.13 & 1182.47 & 1825.79 & 1689 \\
\hline Apr-21 & 1208.56 & 1055.08 & 1562.04 & 1274 \\
\hline May-21 & 929.63 & 746.69 & 1312.58 & \\
\hline Jun-21 & 1102.25 & 891.98 & 1413.32 & \\
\hline Jul-21 & 1176.56 & 939.70 & 1513.60 & \\
\hline Aug-21 & 1134.44 & 972.64 & 1696.66 & \\
\hline Sep-21 & 1024.85 & 838.66 & 1410.65 & \\
\hline Oct-21 & 970.60 & 761.61 & 1379.69 & \\
\hline Nov-21 & 977.12 & 746.41 & 1308.89 & \\
\hline Dec-21 & 1065.08 & 812.97 & 1318.33 & \\
\hline
\end{tabular}

\section{Figures}

FIGURE 1 MEAN AND SPREAD OF THE DATA WITH AND WITHOUT OUTLIER
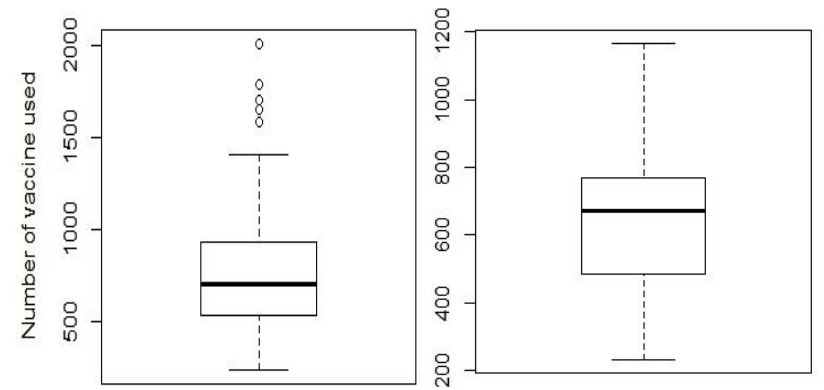

FIGURE 2 ACF AND PACF PLOT TO DETERMINE THE ORDER OF P AND Q
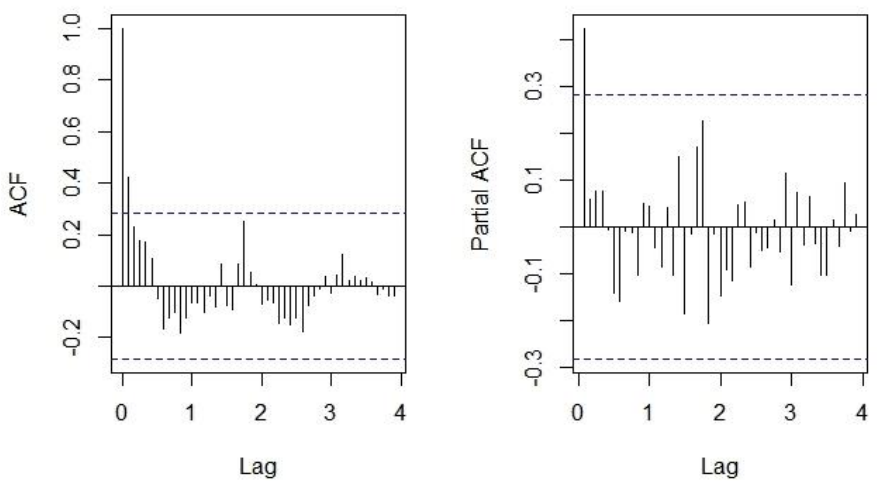

FIGURE 3 FITTED AND FORECASTED VALUE FOR ANTI RABIES VACCINES BASED ON ARIMA $(0,1,1)$ MODEL

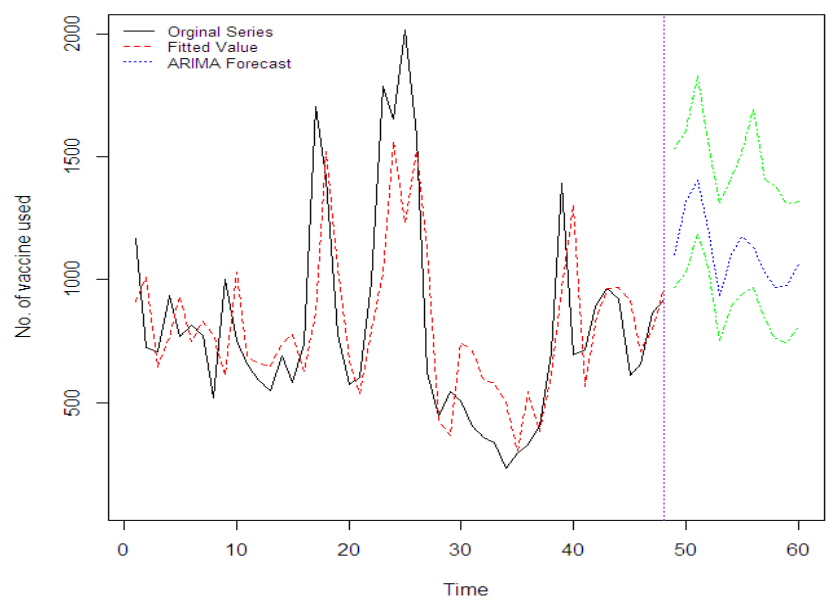

